\title{
ENERGY FUNCTIONAL OF ROTATIONALLY SYMMETRIC HARMONIC MAPS FROM A BALL INTO A SPHERE
}

\author{
Chriestie E.J.C. Montolalu ${ }^{1)}$ \\ ${ }^{1)}$ Program Studi Matematika FMIPA, Universitas Sam Ratulangi Manado \\ e-mail: chriestelly@yahoo.com
}

\begin{abstract}
A study in Rotationally Symmetric Harmonic Maps has been conducted in the past few decades. One of its well-known study is its application from a ball into a sphere in three dimensional space. This has been shown to be accurate by showing its energy function. This paper will show how to find an energy function for this case.
\end{abstract}

Keywords: Rotationally Symmetric Harmonic Maps, Energy Functional

\section{FUNGSI ENERGI DARI PEMETAAN ROTASI HARMONIK SIMETRIS DARI BOLA KE SPHERE}

\begin{abstract}
ABSTRAK
Studi tentang Pemetaan Rotasi Harmonik yang simetris telah dilakukan selama beberapa dekade terakhir ini. Salah satunya yang paling dikenal adalah aplikasinya dari bola ke sphere dalam ruang dimensi tiga. Hal ini telah dapat ditunjukkan dengan akurat dengan membuktikan fungi energinya. Tulisan ini akan menunjukkan cara menemukan fungsi energi untuk masalah ini.
\end{abstract}

Kata-kata kunci: Pemetaan Rotasi Harmonik Simetris, Fungsi Energi

\section{Introduction}

An object with rotational symmetry is an object that looks the same after a certain amount of rotation. A map between two compact Riemannian manifolds is a harmonic map if it is a critical point for the energy functional. For example, a map from a circle to the equator of standard 2-sphere is a harmonic map, and so are the maps that take the circle and map it around the equator $n$ times, for any integer $n$.

Let $M$ be a $n$-dimensional Riemannian manifold (with or without boundary) with a smooth Riemannian metric $g$. In a local coordinates around fixed point $p \in M, g$ can be represented by

$$
g=g_{i j} d x_{i} \otimes d x_{j}
$$

where $g_{i j}$ is a positive definite symmetric $n \times n$ matrix. Let $\left(g^{i j}\right)=\left(g_{i j}\right)^{-1}$ be the inverse matrix of $\left(g_{i j}\right)$ and the volume element of $(M ; g)$ is

$$
d v_{g}=\sqrt{|g|} d x
$$

where $|g|=\operatorname{det}\left(g_{i j}\right)$. Let $N$ be another $l$ dimensional compact Riemmanian manifold (without boundary) with a smooth Riemmanian metric $h$.

For a map $u: M \rightarrow N$, its Dirichlet energy functional is defined by

$$
E(u)=\int_{M} e(u) d v_{g}
$$

where the density function $e(u)$ is given by

$$
\begin{aligned}
& e(u)(x)=\frac{1}{2}|\nabla u(x)|^{2} \\
& =\frac{1}{2} \sum_{\alpha, \beta, i, j} g^{i j}(x) h_{\alpha \beta}(u(x)) \frac{\partial u^{\alpha}}{\partial x_{i}} \frac{\partial u^{\beta}}{\partial x_{j}}
\end{aligned}
$$

In this case, the harmonic map was considered to be from unit ball to unit sphere which satisfies a variational problem in Euler equation form:

$-\Delta u=|\nabla u|^{2} . u$.

Let $\kappa \geq 0$ be an upper bound for the sectional curvature of $N$ and $K_{p}(q)$ the open geodesic ball in $N$ with center $q$ and radius $\rho$. Assuming essentially the size restriction 
$f(\partial M) \subset K_{p}(q), \rho \leq \frac{\pi}{2 \sqrt{\kappa}}$,

Hilderbrandt et. Al. [4] showed existence of a "small" smooth harmonic maps satisfying (1.2). This was shown by considering solution of a Dirichlet problem, which is smooth in the interior and minimizes the energy in the class in $K_{p}(q)$ having the boundary values $f$. In the case where $N$ is the standard sphere the smallness condition restricts the image of the boundary values and the solution to an open half-sphere.

Suppose $B^{n}$ denotes the compact unit ball in the Euclidean space $R^{n}$, and $S^{n}$ the unit sphere in $R^{n+1}$. Select the point given by the $(n+1)$-th standard base vector $e_{n+1}$ in $R^{n+1}$ as northpole of the sphere. Every map $u: B^{n} \rightarrow S^{n}$ can be written in the form $u(x)=(g(x) \cdot \sin \varphi(x), \cos \varphi(x))$

(1.3)

with maps

$$
\varphi: B^{n} \rightarrow[0, \pi], \quad g: B^{n} \rightarrow S^{n-1} \subset R^{n}
$$

$\varphi$ measures the Riemannian distance from $u(x)$ to the northpole on the sphere and is called radius function of $u$. The map $g$ is uniquely defined by $u$ except for points $x$ where $u(x)= \pm e_{n+1}$. A map $u: B^{n} \rightarrow S^{n}$ is rotationally symmetric if and only if

$g(x)=\frac{x}{|x|}$ and $\varphi(x)=\Phi(|x|)$

\section{Notation and Definition}

\section{Euclidean Scalar product and norm}

For the (column) vectors:

$\boldsymbol{u}=\left(u_{1}, u_{2}, \ldots u_{n}\right)^{T}$ and

$\boldsymbol{v}=\left(v_{1}, v_{2}, \ldots v_{n}\right)^{T}$ lying in the Euclidean

space $\mathrm{R}^{n}$, the scalar (dot) product between $\boldsymbol{u}$ and $\boldsymbol{v}$ is:

$$
\begin{gathered}
\langle\boldsymbol{u}, \boldsymbol{v}\rangle=\boldsymbol{u} \cdot \boldsymbol{v} \\
=u_{1} v_{1}+u_{2} v_{2}+\cdots+u_{n} v_{n}=\sum_{i=1}^{n} u_{i} v_{i}
\end{gathered}
$$

and the scalar product of a vector to itself:

$$
\langle\boldsymbol{v}, \boldsymbol{v}\rangle=\boldsymbol{v} \cdot \boldsymbol{v}=v_{1}^{2}+v_{2}^{2}+\cdots+v_{n}^{2}=\sum_{i=1}^{n} v_{i}^{2}
$$

Consequently, the Euclidean norm of a vector is found by taking the square root:

$$
\begin{aligned}
\|\boldsymbol{v}\|=\sqrt{\boldsymbol{v} \cdot \boldsymbol{v}} & =\sqrt{v_{1}^{2}+v_{2}^{2}+\cdots+v_{n}^{2}} \\
& =\left(\sum_{i=1}^{n} v_{i}^{2}\right)^{\frac{1}{2}}
\end{aligned}
$$

\section{Weak Derivative}

Consider the following notation:

$$
\begin{gathered}
x=\left(x_{1}, x_{2}, \ldots, x_{n}\right) \in R^{n}, \quad \partial_{j} u=\frac{\partial u}{\partial x_{j}} \\
\alpha=\left(\alpha_{1}, \alpha_{2}, \ldots, \alpha_{n}\right) \in Z^{n}, \quad \text { is a multi index } \\
|\alpha|=\alpha_{1}+\alpha_{2}+\cdots+\alpha_{n}, \quad \partial^{\alpha} u \\
=\frac{\partial^{|\alpha|}}{\partial x_{1}^{\alpha_{1}} \partial x_{2}^{\alpha_{2}} \ldots \partial x_{n}^{\alpha_{n}}} \\
\nabla u=\left(\partial_{1} u, \ldots, \partial_{n} u\right), \quad|\nabla u| \\
=\left(\sum_{j=1}^{n}\left|\partial_{j} u\right|^{2}\right)^{\frac{1}{2}}
\end{gathered}
$$

Definition 3 (Weak Derivative) [4]

Let $\alpha$ be a multi-index. Suppose that $u, v \in C_{0}^{\infty}(\Omega)$ and

$$
\begin{aligned}
& \int_{\Omega} u(x) \partial^{\alpha} \eta(x) d x \\
& =(-1)^{|\alpha|} \int_{\Omega} v(x) \eta(x) d x, \forall \eta \in C_{0}^{\infty}(\Omega)
\end{aligned}
$$

Then $v$ is called the weak partial derivative of $u$ in $\Omega$, and is denoted by $\partial^{\alpha} u$.

Sobolev spaces $W_{p}^{l}(\Omega)$ and $W_{p}^{l}(\Omega)$

Definition 4 (definition of $W_{p}^{l}(\Omega)$ ) [2]

Suppose that $u \in L_{p}(\Omega)$ and there exist weak derivatives $\partial^{\alpha} u$ for any $\alpha$ with $|\alpha| \leq l$ (all derivatives up to order $l$ ), such that

$$
\partial^{\alpha} u \in L_{p}(\Omega),|\alpha| \leq l .
$$

Then we say that $u \in W_{p}^{l}(\Omega)$.

The standard norm in $W_{p}^{l}(\Omega)$ :

$$
|u|_{W_{p}^{l}(\Omega)}=\left(\int_{\Omega} \sum_{|\alpha| \leq l} \partial^{\alpha} u^{p} d x\right)^{\frac{1}{p}}
$$

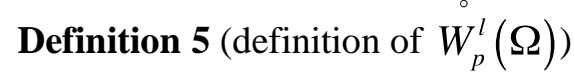

The closure in the norm of $W_{p}^{l}(\Omega)$ is denoted 
by $\stackrel{\circ}{W_{p}^{l}}(\Omega)$.

So, $W_{p}^{l}(\Omega)$ is a subspace in the space $W_{p}^{l}(\Omega)$.

For $=2, W_{2}^{l}(\Omega)$, another notation $H^{l}(\Omega)$ is often used: $W_{2}^{l}(\Omega)=H^{l}(\Omega)$.

\section{Energy Functional}

For $B^{n}$ denotes the compact unit ball in the Euclidean space $R^{n}$, and $S^{n}$ the unit sphere in $R^{n+1}$, the subset of mappings of the Sobolev space $H^{1}\left(B^{n}, R^{n+1}\right)$, satisfying $u(x) \in$ $S^{n}, \quad \forall x \in B^{n} \quad$ is denoted by $\mathscr{S}^{n}=H^{1}\left(B^{n}, S^{n}\right)[2]$.

For $\quad u, v \in H^{1}\left(B^{n}, R^{n+1}\right) \quad$ the weak derivative, $\partial^{\alpha}$, can be denoted in the Euclidean scalar and norm as follows:

$\langle\nabla u, \nabla v\rangle=\sum_{\alpha=1}^{n}\left\langle\partial^{\alpha} u, \partial^{\alpha} v\right\rangle$, and $|\nabla u|=$ $\langle\nabla u, \nabla v\rangle^{\frac{1}{2}}$

The Dirichlet energy functional for $u \in \mathscr{S}^{n}$ is defined by:

$$
E(u)=E_{n}(u)=\frac{1}{2} \int_{B^{n}}|\nabla u|^{2} d x
$$

\section{Harmonic Maps}

Recall that a Laplace equation on an open domain $\Omega$ of $R^{n}$ is defined by

$$
\begin{gathered}
\Delta u=0 \\
\text { where } \\
\Delta=\frac{\partial^{2}}{\left(\partial x_{1}\right)^{2}}+\cdots+\frac{\partial^{2}}{\left(\partial x_{n}\right)^{2}},\left(x_{1}, \ldots, x_{n}\right) \in \Omega
\end{gathered}
$$

In this project, we consider a variational problem with Euler equation:

$$
-\Delta u=|\nabla u|^{2} \cdot u \text {. }
$$

Definition 6 (Harmonic Map) [3,5]

A smooth map $u$ from $B^{n}$ to $S^{n}$ is said to be harmonic map if $u$ is a critical point of the Dirichlet energy functional (2.1), i.e. it satisfies (2.2).

To see the harmonic map in this project, consider the following problem.

Dirichlet problem. Given boundary values $f: \partial B^{n} \rightarrow S^{n}$ of class $H^{\frac{1}{2}}$. Find a critical point $u$ of the Energy $E$ in the class of mappings in $\mathrm{S}^{n}$, satisfying $\left.u\right|_{\partial B^{n}}=f$.

The critical point can be obtained as follows: First denote for each $u \in S^{n}$, the space of vector-fields along $u$, by

$$
\mathscr{S}^{n}=\left\{v \in H^{1}\left(B^{n}, R^{n+1}\right):\langle u(x), v(x)\rangle=0, \forall x \in B^{n}\right\}
$$

and set

$$
\delta_{u} \mathscr{S}_{0}^{n}=\stackrel{\circ}{H^{1} \cap} \delta_{u} \mathscr{\rho}^{n},
$$

where $\stackrel{\circ}{H}^{1}$ is the closure (subspace) of $H^{1}$.

Critical point of $E$ can be obtained by setting the first variation of $E$ to zero. Following that, the stability of this critical point can be determined by considering the second variation of $E$.

First and second variation of $E(v)=\frac{1}{2} \int_{B^{n}}|\nabla v|^{2} d x$ can be calculated as follows:

For $t$ small, define $u_{t}: B^{t} \rightarrow S^{n}$ by setting:

$$
u_{t}(x)=\frac{u+t v}{\left(1+t^{2} v^{2}\right)^{1 / 2}}
$$

Thus,

$$
\nabla u_{t}=\frac{\nabla u+t \nabla v}{\left(1+t^{2} v^{2}\right)^{1 / 2}}-\frac{1}{2} \frac{t^{2}(u+t v) \nabla v^{2}}{\left(1+t^{2} v^{2}\right)^{3 / 2}}
$$

Note that:

$$
\begin{gathered}
\frac{\partial}{\partial t}\left|\nabla u_{t}\right|^{2}=2 \nabla u_{t} \cdot\left(\frac{\partial}{\partial t} \nabla u_{t}\right) \\
\text { and } \\
\frac{\partial^{2}}{\partial t^{2}}\left|\nabla u_{t}\right|^{2}=2\left[\left(\frac{\partial}{\partial t} \nabla u_{t}\right) \cdot\left(\frac{\partial}{\partial t} \nabla u_{t}\right)+\nabla u_{t} \cdot\left(\frac{\partial^{2}}{\partial t^{2}} \nabla u_{t}\right)\right]
\end{gathered}
$$

Evaluating at $t=0$ gives

$$
\left.\frac{\partial}{\partial t}\left|\nabla u_{t}\right|^{2}\right|_{t=0}=2 \nabla u . \nabla v
$$

and 


$$
\begin{aligned}
\left.\frac{\partial^{2}}{\partial t^{2}}\left|\nabla u_{t}\right|^{2}\right|_{t=0} & =2\left[\nabla v \cdot \nabla v+\nabla u \cdot\left(-\left.\nabla u \cdot v\right|^{2}-u \cdot \nabla|v|^{2}\right)\right] \\
& =2\left[|\nabla v|^{2}-|\nabla u|^{2} \cdot|v|^{2}-\nabla u \cdot u \cdot \nabla|v|^{2}\right]
\end{aligned}
$$

Since $\nabla|v|^{2}=\nabla(v \cdot v)=2 \cdot v \cdot \nabla v$, and $u \cdot v=0$, then $\quad \nabla u \cdot u \cdot \nabla|v|^{2}=\nabla u \cdot 2 \cdot u \cdot v \cdot \nabla v=0 \quad$ and therefore $\left.\frac{\partial^{2}}{\partial t^{2}}\left|\nabla u_{t}\right|^{2}\right|_{t=0}=2\left[|\nabla v|^{2}-|\nabla u|^{2} \cdot|v|^{2}\right]$ Thus, the first variation of

$$
\begin{aligned}
E(v)=\frac{1}{2} \int_{B^{n}}|\nabla v|^{2} d x \text { is } \\
\begin{aligned}
\delta_{u} E(v)=\left.\frac{\partial}{\partial t}\right|_{t=0} E\left(u_{t}\right) & =\left.\frac{1}{2} \int_{B^{n}} \frac{\partial}{\partial t}\left|\nabla u_{t}\right|^{2}\right|_{t=0} d x \\
& =\int_{B^{n}}\langle\nabla u, \nabla v\rangle d x
\end{aligned}
\end{aligned}
$$

and the second variation is

$$
\begin{aligned}
\delta_{u}{ }^{2} E(v) & =\left.\frac{\partial^{2}}{\partial t^{2}} E\left(\nabla u_{t}\right)\right|_{t=0}=\left.\frac{1}{2} \int_{B^{n}} \frac{\partial^{2}}{\partial t^{2}}\left|\nabla u_{t}\right|^{2}\right|_{t=0} d x \\
& \left.=\left.\int_{B^{n}}|| \nabla v\right|^{2}-|\nabla u|^{2} \cdot|v|^{2}\right] d x
\end{aligned}
$$

By setting the first variation to be equal to zero, then $u$ can be a harmonic map of $E$ if it satisfies

$$
\delta_{u} E(v)=\int_{B^{n}}\langle\nabla u, \nabla v\rangle d x=0
$$

and it is stable if $\delta_{u}{ }^{2} E(v) \geq 0$.

By definition 6, $u$ must satisfy (2.2). We will see that (2.3) is a weak form of (2.2).

Suppose a vector $w, w \in \dot{\circ}_{2}^{1}\left(B^{n}, R^{n+1}\right)$, such that the component vector $v$ can be written as component of $w$ orthogonal to $u$, (since $\langle u(x), v(x)\rangle=0$ ) as follows

$$
\begin{gathered}
v=w-\operatorname{proj}_{u}(w), \\
\text { where } \operatorname{proj}_{u}(w)=\frac{\langle u, w\rangle}{|u|^{2}} u,
\end{gathered}
$$

$|u|^{2}=1$ since $u(x) \in S^{n}$, where $S^{n}$ is a unit sphere.

then $v=w-\langle u, w\rangle u$.

Substituting this into $\delta_{u} E(v)$ yields

$$
\begin{aligned}
\delta_{u} E(v) & =\int_{B^{n}}\langle\nabla u, \nabla v\rangle d x \\
& =\int_{B^{n}}\langle\nabla u, \nabla(w-\langle u, w\rangle u)\rangle d x \\
& =\int_{B^{n}}\langle\nabla u, \nabla w-\nabla(\langle u, w\rangle u)\rangle d x \\
& =\int_{B^{n}}[\langle\nabla u, \nabla w\rangle-\langle\nabla u,(\nabla\langle u, w\rangle u+\langle u, w\rangle \nabla u)\rangle] d x \\
& =\int_{B^{n}}[\langle\nabla u, \nabla w\rangle-\langle\nabla u,\langle u, w\rangle \nabla u\rangle-\langle\nabla u,(\nabla\langle u, w\rangle u)\rangle] d x \\
& =\int_{B^{n}}\left[\langle\nabla u, \nabla w\rangle-|\nabla u|^{2}\langle u, w\rangle\right] d x-\int_{B^{n}}[u \nabla u(\nabla\langle u, w\rangle)] d x
\end{aligned}
$$

Since $w$ is zero on the boundary and

$$
-\Delta u=|\nabla u|^{2} \cdot u \Rightarrow u \Delta u+|\nabla u|^{2}=0 \text { then }
$$

$$
\begin{aligned}
\int_{B^{n}} u \nabla u(\nabla\langle u, w\rangle) d x \quad & =\left.u \nabla u\langle u, w\rangle\right|_{B^{n}}-\int_{B^{n}}\langle u, w\rangle \nabla(u \nabla u) d x \\
& =0-\int_{B^{n}}\langle u, w\rangle[\nabla u \nabla u+u \nabla(\nabla u)] d x \\
& =-\int_{B^{n}}\langle u, w\rangle\left[|\nabla u|^{2}+u \Delta u\right] d x \\
& =0
\end{aligned}
$$

so,

$$
\int_{B^{n}}\langle\nabla u, \nabla v\rangle d x=\int_{B^{n}}\left\langle\langle\nabla u, \nabla w\rangle-|\nabla u|^{2}\langle u, w\rangle\right| d x=0
$$

This is weak solution of $\Delta u+|\nabla u|^{2} \cdot u=0$, which can be shown as follows.

Suppose a vector $w, w \in \stackrel{\circ}{H}_{2}^{1}\left(B^{n}, R^{n+1}\right)$

$$
\int_{B^{n}}-\Delta u \cdot w d x=\int_{B^{n}}|\nabla u|^{2}\langle u, w\rangle d x
$$

It is clear that

$$
\begin{aligned}
\int_{B^{n}}-\Delta u \cdot w d x & =\int_{B^{n}}-\nabla(\nabla u) \cdot w d x \\
& =-\left.\nabla u \cdot w\right|_{B^{n}}+\int_{B^{n}} \nabla u \cdot \nabla w d x \\
& =0+\int_{B^{n}} \nabla u \cdot \nabla w d x \\
& =\int_{B^{n}} \nabla u \cdot \nabla w d x
\end{aligned}
$$


Hence, it can be shown that

$$
\begin{gathered}
\int_{B^{n}}\langle\nabla u, \nabla w\rangle d x=\int_{B^{n}}|\nabla u|^{2}\langle u, w\rangle d x \\
\text { or } \\
\int_{B^{n}}\left\langle\langle\nabla u, \nabla w\rangle-|\nabla u|^{2}\langle u, w\rangle\right| d x=0
\end{gathered}
$$

is the weak solution of $-\Delta u=|\nabla u|^{2} \cdot u$

Therefore $u$ is a harmonic map if it satisfies the weak solution of

Consider the map

$$
-\Delta u=|\nabla u|^{2} \cdot u
$$

$$
u_{*}: B^{n} \rightarrow S^{n}, x \rightarrow\left(\frac{x}{|x|}, 0\right)
$$

where $u_{*}$ belongs to $S^{n}$ for $n \geq 3$ and is a weak solution of $-\Delta u=|\nabla u|^{2}$. $u$, then $u_{*}$ is called the equator map. The well-known remark of Heinz [2], pointing out that a size restriction is necessary in order to bound the derivates of the solutions to certain elliptic systems, is using solutions of equation (2.6).

\section{Rotationally Symmetric Harmonic Maps}

We are going to study rotationally symmetric maps with finite energy.

Recall that every map $u: B^{n} \rightarrow S^{n}$ can be written in the form

$u(x)=(g(x) \cdot \sin \varphi(x), \cos \varphi(x))$

with maps

$$
\varphi: B^{n} \rightarrow[0, \pi], g: B^{n} \rightarrow S^{n-1} \subset R^{n}
$$

$\varphi$ measures the Riemannian distance from $u(x)$ to the northpole on the sphere and is called radius function of $u$. A map $u: B^{n} \rightarrow$ $S^{n}$ is rotationally symmetric if and only if

$$
g(x)=\frac{x}{|x|} \text { and } \varphi(x)=\Phi(|x|)
$$

In this case, metric form of the manifold can be written as:

$$
|u|^{2}=|\varphi|^{2}+f^{2}(\varphi) \cdot|g|^{2}
$$

Since the projection assumed is projection from unit ball to unit sphere, then this can be assumed as a function in geodesic coordinate of a sphere, i.e. $f(\varphi)=\sin \varphi$.

Therefore, for

$u(x)=\left(\frac{x}{r} \sin \Phi(r), \cos \Phi(r)\right)$,

with radius function $\Phi:[0,1] \rightarrow[0, \pi]$ depending only on $r=|x|$, the metric form can be written as and

$$
|u|^{2}=|\Phi(r)|^{2}+\sin ^{2}(\Phi(r)) \cdot\left|\frac{x}{r}\right|^{2}
$$

$$
|\nabla u|^{2}=|\nabla \Phi(r)|^{2}+\sin ^{2}(\Phi(r)) \cdot\left|\nabla\left(\frac{x}{r}\right)\right|^{2}
$$

It is clear that

$$
\begin{aligned}
|\nabla \Phi(r)|^{2} & =\sum_{\alpha=1}^{n}\left|\Phi^{\prime}(r) \frac{\partial r}{\partial x^{\alpha}}\right|^{2} \\
& =\left(\Phi^{\prime}(r)\right)^{2} \sum_{\alpha=1}^{n}\left|\frac{\partial|x|}{\partial x^{\alpha}}\right|^{2} \\
& =\left(\Phi^{\prime}(r)\right)^{2} \sum_{\alpha=1}^{n}\left|\frac{x^{\alpha}}{|x|}\right|^{2} \\
& =\Phi^{\prime 2}(r) \\
\frac{\partial}{\partial x^{\alpha}}\left(\frac{x}{r}\right) & =\frac{\partial x_{i}}{\partial x^{\alpha}} \cdot \frac{1}{r}+x \frac{\partial_{i}}{\partial x^{\alpha}}\left(\frac{1}{r}\right) \\
& =\frac{r^{2} \frac{\partial x_{i}}{\partial x^{\alpha}}-x \cdot x_{i}}{r^{3}}
\end{aligned}
$$

Then

$$
\begin{aligned}
\left|\nabla\left(\frac{x}{r}\right)\right|^{2} & =\sum_{1 \leq \alpha, i \leq n}\left|\frac{\partial}{\partial x^{\alpha}}\left(\frac{x}{r}\right)\right|^{2} \\
& =\sum_{1 \leq \alpha, i \leq n}\left|\frac{r^{2} \frac{\partial x_{i}}{\partial x^{\alpha}}-x \cdot x_{i}}{r^{3}}\right|^{2} \\
& =\left[\frac{r^{4}\left(\sum_{i=1}^{n} 1\right)-2 r^{2} \cdot r^{2}+r^{4}}{r^{6}}\right] \\
& =\left[\frac{n r^{4}-r^{4}}{r^{6}}\right] \\
& =\frac{n-1}{r^{2}}
\end{aligned}
$$

and therefore

$|\nabla u|^{2}=\Phi^{\prime 2}(r)+\frac{n-1}{r^{2}} \sin ^{2} \Phi(r)$

Thus

$E(u)=\frac{1}{2} \int_{B^{n}}\left[\Phi^{\prime 2}(r)+\frac{n-1}{r^{2}} \sin ^{2} \Phi(r)\right] d x$ and since the Volume of $(n-1)$-dimensional unit sphere

$$
V_{S}^{n-1} r^{n-1}=n V_{B}^{n} r^{n-1},
$$

then suppose $\omega_{n}$ denotes the volume of $n$ dimensional unit ball. 


$$
\begin{aligned}
& E(u) \\
& =\frac{1}{2} \int_{0}^{1} \int_{0}^{\pi}\left[\Phi^{\prime 2}(r)\right. \\
& \left.+\frac{n-1}{r^{2}} \sin ^{2} \Phi(r)\right] d \theta r^{n-1} d r
\end{aligned}
$$

$E(u)=$

$\frac{\omega_{n}}{2} \int_{0}^{1}\left[\Phi^{\prime 2}+\right.$

$$
\left.\frac{n-1}{r^{2}} \sin ^{2} \Phi(r)\right] r^{n-1} d r
$$

The first variation now becomes

$$
\begin{aligned}
\delta_{u} E(v)=\int_{B^{n}} \nabla & \nabla \cdot \nabla v \\
& +\sin \Phi \cdot \cos \Phi \cdot v \cdot\left|\nabla\left(\frac{x}{r}\right)\right|^{2} \\
& +\sin ^{2} \Phi \cdot \nabla\left(\frac{x}{r}\right) \cdot \nabla \xi d x
\end{aligned}
$$

For $\mathrm{v} \in \delta_{u} \mathrm{~S}_{0}^{n}$, where $v=|\mathrm{v}|$ and $\xi=\frac{\mathrm{v}}{|\mathrm{v}|}$.

Since $\Delta\left(\frac{x}{r}\right)=0$, then

$\int_{B^{n}} \nabla\left(\frac{x}{r}\right) \cdot \nabla \xi d x=\int_{B^{n}}-\Delta\left(\frac{x}{r}\right) \cdot \nabla \xi d x=0$

And since $\delta_{u} E(v)=0$, thus

$\int_{B^{n}} \nabla \Phi . \nabla v d x$

$=-\int_{B^{n}} \sin \Phi \cdot \cos \Phi \cdot v \cdot\left|\nabla\left(\frac{x}{r}\right)\right|^{2} d x$

$=-\int_{B^{n}} \frac{n-1}{r^{2}} \sin \Phi \cdot \cos \Phi \cdot v \cdot d x$

And since $\int_{B^{n}} \nabla \Phi \cdot \nabla v d x=-\int_{B^{n}} \Delta \Phi \cdot v d x$, then

$\int_{B^{n}} \Delta \Phi \cdot v d x$

$=\int_{B^{n}} \frac{n-1}{r^{2}} \sin \Phi \cdot \cos \Phi \cdot v \cdot d x$

Thus,

$\Delta \Phi=\frac{n-1}{r^{2}} \sin \Phi \cdot \cos \Phi=\frac{n-1}{2 r^{2}} \sin 2 \Phi$

In $n$ dimensions the spherical satisfies the partial differential equation

$$
\Delta \Phi(r)=\frac{1}{r^{n-1}} \frac{\partial}{\partial r} r^{n-1} \frac{\partial}{\partial r} \Phi(r)
$$

This can be expressed as : $\frac{1}{r^{n-1}} \frac{\partial}{\partial r} r^{n-1} \frac{\partial}{\partial r} \Phi(r)=\frac{\partial^{2}}{\partial r^{2}} \Phi(r)+$ $\frac{n-1}{r} \frac{\partial}{\partial r} \Phi(r)$.

Thus

$$
\begin{gathered}
\frac{\partial^{2}}{\partial r^{2}} \Phi(r)+\frac{n-1}{r} \frac{\partial}{\partial r} \Phi(r)-\frac{n-1}{2 r^{2}} \sin 2 \Phi \\
=0 \\
\Phi^{\prime \prime(r)}+\frac{n-1}{r} \Phi^{\prime(r)}-\frac{n-1}{2 r^{2}} \sin 2 \Phi=0
\end{gathered}
$$

Suppose $\quad \Psi(t)=\Phi\left(e^{t}\right), \quad \Psi:(-\infty, 0] \rightarrow$ $[0, \pi]$

$\Psi^{\prime}(t)=e^{t} \Phi^{\prime}\left(e^{t}\right)$

$\Psi^{\prime \prime}(t)=e^{t} \Phi^{\prime}\left(e^{t}\right)+e^{2 t} \Phi^{\prime \prime}\left(e^{t}\right)$

Then

$$
\begin{gathered}
\begin{aligned}
\Phi^{\prime \prime}\left(e^{t}\right)+\frac{n-1}{e^{t}} & \Phi^{\prime}\left(e^{t}\right)-\frac{n-1}{2 e^{2 t}} \sin 2 \Phi\left(e^{t}\right) \\
& =0
\end{aligned} \\
\begin{aligned}
& e^{2 t} \Phi^{\prime \prime}\left(e^{t}\right)+(n-1) e^{t} \Phi^{\prime}\left(e^{t}\right) \\
&-\frac{n-1}{2} \sin 2 \Phi\left(e^{t}\right)=0 \\
& {\left[e^{2 t} \Phi^{\prime \prime}\left(e^{t}\right)+e^{t}\right.}\left.\Phi^{\prime}\left(e^{t}\right)\right] \\
&+(n-2)\left[e^{t} \Phi^{\prime}\left(e^{t}\right)\right] \\
&-\frac{n-1}{2} \sin 2 \Phi\left(e^{t}\right)=0
\end{aligned}
\end{gathered}
$$

Therefore,

$$
\Psi^{\prime \prime}(t)+(n-2) \Psi^{\prime}(t)-\frac{n-1}{2} \sin 2 \Psi(t)=
$$

The energy can be expressed in term of $\Psi(t)$ :

$E(u)$

$$
\begin{aligned}
& =\frac{\omega_{n}}{2} \int_{-\infty}^{0}\left[\Phi^{\prime 2}\left(e^{t}\right)\right. \\
& \left.+\frac{n-1}{e^{2 t}} \sin ^{2} \Phi\left(e^{t}\right)\right] e^{(n-1) t} e^{t} d t \\
& =\frac{\omega_{n}}{2} \int_{-\infty}^{0}\left[e^{2 t} \Phi^{\prime 2}\left(e^{t}\right)\right. \\
& \left.+(n-1) \sin ^{2} \Phi\left(e^{t}\right)\right] e^{-2 t} e^{(n-1) t} e^{t} d t \\
& =\frac{\omega_{n}}{2} \int_{-\infty}^{0}\left[\Psi^{\prime 2}(t)+(n-\right. \\
& \left.1) \sin ^{2} \Psi(t)\right] e^{(n-2) t} d t
\end{aligned}
$$

\section{References}

[1] E. Heinz, On certain nonlinear elliptic differential equations and univalent 
mappings, $J$. d'Analyse Mathematique. 5(1956/1957) 197-192.

[2] L.C. Evans, Partial differential equations, American Mathematical Society 2010.

[3] M. Hong, Some results on harmonic maps, Academia Sinica. 9(2014) 187-221.
[4] P. Hartman, Ordinary differential equations, New York 1964.

[5] S. Hilderbrandt, H. Kaul, and K. O. Widman, An existence theorem for harmonic mappings of Riemmanian manifolds, Acta. Math. 138(1977), 1-16. 